\title{
Análise teórica de itens de uma escala americana para avaliação do atendimento em creches inclusivas brasileiras
}

\begin{abstract}
Lisandrea Rodrigues Menegasso Gennaro* Maria Stella Coutinho de Alcântara Gi**

Resumo

Com a proposta de uma educação infantil inclusiva, são necessários mecanismos para avaliar a qualidade da educação infantil para crianças com necessidades educacionais especiais. A escala Infant/Toddler Environment Rating Scale Revised avalia a qualidade de ambientes coletivos de educação infantil sob diferentes aspectos como ambiente físico, interação, atividades e atendimento às crianças com necessidades especiais. Estudos vêm investigando a viabilidade da ITERS-R para o contexto brasileiro. Na direção destes estudos, a pesquisa teve como objetivo verificar a compreensão dos termos e a pertinência do conteúdo dos itens da Infant/Toddler Environment Rating Scale - Revised Edition, relacionados a pessoas com deficiência. Foram identificados e traduzidos 12 itens da escala que se referem às necessidades educacionais especiais por especialista bilingüe. Os itens foram submetidos à análise de conteúdo por sete especialistas em educação infantil e educação especial, para que avaliassem a pertinência dos itens, e à análise semântica por sete alunos do curso de psicologia, por meio de registro em protocolos de respostas. Os dados foram transcritos e analisados de maneira qualitativa. Os resultados indicaram que os itens são pertinentes e apontam caminhos para torná-los mais acurados para avaliação de creches brasileiras. Conclui-se que os itens analisados podem contribuir para a promoção e avaliação da qualidade em creches, em relação às pessoas com deficiência, ainda que haja a necessidade de aprimorar a acurácia do conteúdo dos itens.
\end{abstract}

Palavras-chave: Creche; Avaliação de qualidade; Escala ITERS-R.

\footnotetext{
* Psicóloga na Universidade Federal de São Carlos. Doutoranda no Programa de Pós-Graduação em Psicologia da UFSCar. São Calos, São Paulo.

** Professora Doutora da Universidade Federal de São Carlos, Centro de Educação e Ciências Humanas, Departamento de Psicologia. São Carlos, São Paulo.
} 


\title{
Theoretical analysis of items from an American scale for assessment of inclusive kindergartens in Brazil
}

\begin{abstract}
With the proposal of an inclusive early childhood education, it is necessary mechanisms to evaluate the quality of the education for infants and toddlers with special needs. The Infant/Toddler Environment Rating Scale Revised was developed to evaluate the quality of collective environments of child education under different aspects as surrounding physicist, interaction, activity and provisions to the children with special needs. Studies are investigating the viability of the scale for Brazilian context. In the direction of these studies, the research had as objective verify the understanding and the relevancy of the content of itens of the Infant/Toddler Environment Rating Scale - Revised Edition that deal with people with disabilities. It had been identified and translated 12 itens of the scale by a bilingual specialist. Then, it had been submitted to the content analysis for seven female specialists in child and special education, so that they evaluated the relevancy of itens, and to the semantic analysis for seven pupils of the course of psychology so that they evaluated the clearly and the understanding of itens, in both the cases registering in protocols. The data had been transcribed and analyzed in a qualitative way. The results had indicated that itens are pertinents and points ways to increase the accuracy with respect to future studies. Concludes that itens of the scale analyzed can contribute for the promotion and evaluation of the quality in early childhood centers but it is still necessary to work towards improve the accuracy of the content of itens.
\end{abstract}

Keywords: Infant educational environments; Evaluation of quality; Scale ITERS-R.

Introdução

Os educadores de alunos com necessidades educacionais especiais concordam que a educação deve ser iniciada desde a mais tenra idade, com atendimento de caráter educativo, superando o antigo conceito de educação infantil como atendimento de caráter tutelar, orientado para o cuidado com a criança em relação à segurança, higiene e alimentação (MARQUES, 2000). Concordam ainda que as instituições de educação infantil devem proporcionar às crianças com necessidades educacionais especiais uma educação adequada e adaptada à realidade de cada uma delas, conforme consta nas Diretrizes Nacionais para a Educação Especial na Educação Básica (BRASIL, 2001). A falta de atendimento adequado para estas crianças, por falta de preparo dos educadores ou de recursos pode gerar 
atrasos no desenvolvimento assim como privar as crianças de uma educação libertadora. Os princípios fundamentais do paradigma da inclusão afirmam que não basta acolher crianças com necessidades educacionais especiais na creche. É necessário promover a educação e que esta educação faça jus ao potencial próprio do período do desenvolvimento infantil juntamente com crianças de desenvolvimento típico porque as crianças têm igualdade de direitos. Pesquisas como a de Rosa (2003) indicam a presença, na creche, de crianças com necessidades educacionais especiais, especialmente crianças de risco para problemas no desenvolvimento. A autora investigou a incidência de crianças especiais inseridas na rede municipal de creches da cidade de São Carlos. Participaram do estudo atendentes de berçário e diretoras de 12 creches municipais. A identificação das crianças com necessidades especiais se deu por meio de entrevistas e aplicação de um inventário, acompanhado de um manual explicativo das categorias de necessidades educacionais especiais, dirigidos às diretoras e educadoras das creches. O manual apresentava o conceito/definição de sete categorias: Criança de risco; Condutas típicas; Deficiência mental; Deficiência auditiva; Deficiência física; Deficiência múltipla e Altas habilidades. Em uma população total de 1.196 crianças, foram identificadas 102 crianças com necessidades educacionais especiais $(8,5 \%)$ - porcentagem que se aproxima da estimativa da Organização Mundial de Saúde (10\%). Os resultados indicaram que 46\% das crianças foram consideradas Criança de risco, seguido por Condutas típicas $(14,7 \%)$, Deficiência física (5,9\%), Altas habilidades (3,9\%), Mistos (3,9\%), Deficiência Mental (1,9\%) e Deficiência Auditiva e Múltipla (1\%) (as categorias não eram mutuamente exclusivas). As creches estariam acolhendo crianças com necessidades educacionais especiais, principalmente crianças que apresentam risco para problemas no desenvolvimento.

O paradigma de inclusão social constitui-se no fundamento dos avanços legais na educação infantil e na educação especial (ROSSETTIFERREIRA, RAMON; SILVA, 2002). A inclusão social está associada à construção de uma sociedade democrática que reconhece e aceita a diversidade e as diferenças individuais. É um movimento, orquestrado por vários países, que propõe que as pessoas excluídas e a sociedade busquem a equiparação de oportunidades e o exercício da cidadania por todos (MENDES, 2002).

A inclusão na Educação Especial sustenta-se em documentos nacionais oficiais, como a Constituição (BRASIL, 1988), no Estatuto da Criança e do Adolescente (BRASIL, 1990) na Lei de Diretrizes e Bases da Educação Nacional (BRASIL, 1996). Entre os documentos oficiais que dispõem sobre a inclusão em creches, estão o Referencial Curricular Nacional para a Educação Infantil (BRASIL, 1998) e as Diretrizes Nacionais em Educação Especial para a Educação Básica (BRASIL, 2001). 
A vigência do paradigma da inclusão não descarta a existência de uma complexa controvérsia sobre seus aspectos filosóficos, político e de aplicação prática. Por exemplo, para Minto (2002), a inclusão tal como proposta pela LDB permite a adoção de diferentes condutas, contém termos discriminatórios e ambíguos e carece de clareza sobre as responsabilidades do poder público. Para Minto, a LDB não concebe a educação especial com os mesmos objetivos da educação geral; a depender do governo, a educação especial continuará delegada à iniciativa privada porque exime o Estado de assumir suas responsabilidades de assumir e financiar essa complexa modalidade educativa.

Abramowicz (2002) questiona se a inclusão na prática, no cotidiano, na rede regular de ensino, desde a educação infantil até a universidade, vem ocorrendo do modo como está colocada nos diferentes documentos, já que nas leis tudo parece estar em perfeita harmonia para que a inclusão ocorra de modo totalmente satisfatório para todos (deficientes, profissionais, instituições, sociedade, etc.).

Mendes (2002) afirma que faltam estudos sobre as variáveis envolvidas no complexo processo compreendido pela proposta de educação inclusiva. De acordo com levantamento de teses e dissertações sobre Educação Especial, elaborado por Mendes et al. (2001) faltam ainda mais estudos sobre inclusão na educação infantil. De um total de 419 trabalhos, o tema Integração/Inclusão foi abordado em $13,36 \%$ dos trabalhos analisados, sendo que destes, apenas $0,47 \%$ enfocaram a educação para crianças de 0 a 3 anos, ou seja, dois dos 419 trabalhos.

Dahlberg, Moss e Pence (2003), criticam a utilização de padrões de qualidade, indicando uma abordagem mais fluida da discussão sobre qualidade, o que chamam de perspectiva pós-moderna. Criticam o discurso da busca por qualidade na educação infantil por meio de padrões de qualidade, devido ao fato de a educação ser um processo complexo e particular em cada instituição e em cada cultura. A crítica sobre as limitações de padrões e instrumentos para a busca da qualidade do atendimento em instituições de educação infantil é pertinente. No entanto, a postura dos autores de ser "evocativos ao invés de didáticos" ( $p$. 11) pouco contribui, a curto e médio prazo, para alterar a situação, muitas vezes lastimável, em que se encontram as crianças em creches. Piotto et al. (1998) afirmam que cada instituição deveria definir sua qualidade bem como formas de alcançá-la; no entanto, argumenta também que a questão da inviabilidade de padrões pode se constituir num falso paradoxo na medida em que numa sociedade de cultura de massa a diversidade é mais ilusória do que real - as diferenças concretas num mundo globalizado são cada vez menores. 
Para Campos e Rosemberg (2009) a exigência de padrões mínimos visa definir um limite abaixo do qual os serviços apresentam riscos inaceitáveis para as crianças. É importante preservar as condições e características sociais e culturais de cada país ou região, porém não se deve permitir que a população atendida corra riscos de sobrevivência ou não tenha seus direitos básicos respeitados.

Diversos países estão desenvolvendo instrumentos para promoção e avaliação da qualidade em instituições de educação infantil, como a ITERS-R Infant/Toddler Environment Rating Scale- Revised Edition (HARMS, CRYER, CLIFFORD, 2003). A escala ITERS-R permite sistematizar a avaliação de ambientes coletivos de crianças de 0 a 30 meses. A escala torna o conceito de qualidade em educação infantil objetivo porque seleciona e quantifica variáveis relevantes no atendimento da criança em creche. Para medir e definir qualidade, a ITERS-R foi elaborada a partir de três fontes: dados de pesquisas em diferentes áreas, como saúde, desenvolvimento e educação; o que profissionais da área consideravam como melhores práticas no atendimento a crianças de 0 a 30 meses em ambiente coletivos de educação infantil; a prática da vida real no atendimento institucional destas crianças. A escala contém uma parte introdutória, que a apresenta, aborda seu processo de revisão, trata a questão da fidedignidade e validade do instrumento e as referências bibliográficas. Contém também uma parte com instruções para o uso. Nesta seção trata da aplicação da escala, do sistema de pontuação, apresenta uma opção alternativa de pontuação para fins de pesquisa, por exemplo, fornece folhas de pontuação e elaboração do perfil das salas das creches, além de apresentar a descrição de termos que aparecem durante a escala, como "acessível", "apropriado", "maior parte do dia", e outros, para estabelecer critérios para sua utilização.

A escala permite avaliar e promover a qualidade por meio dos 39 itens que estão agrupados em sete sub-escalas: Espaço e mobília, Rotina de cuidados pessoais, Ouvir e Falar, Atividades, Interações, Estrutura do Programa e Pais e Equipe de trabalho. Para avaliar os ambientes existe uma pontuação que varia de 1 a 7 . A pontuação um corresponde ao Inadequado, três ao Mínimo, 5 ao Bom e 7 ao Excelente contendo em cada uma delas uma série de indicadores que compõe os critérios pelos quais os ambientes serão avaliados. Há as pontuações intermediárias 2, 4 e 6 nos casos de o critério de pontuação não ser completamente atingido.

As pontuações variam de acordo com, por exemplo, o controle das condições ambientais como luz, ventilação; condições que facilitam a higiene do ambiente; ambientes e mobílias em bom estado de conservação; espaços adequados para diferentes tipos de atividades e experiências; provisão de 
conforto para adultos e crianças; materiais e equipamentos adequados e em quantidade suficiente; interação calorosa e afetuosa entre a equipe escolar e as crianças, a acessibilidade do espaço físico para permitir equiparação de oportunidades para alunos com necessidades educacionais especiais; a promoção da independência das crianças com a adaptação de locais para higiene e alimentação; a estimulação da cognição, coordenação motora global, fina e linguagem; a promoção da aceitação da diversidade.

De acordo com Harms, Cryer \& Clifford (2003) a ITERS-R é um instrumento abrangente, acessível e válido - para a cultura americana - que pode assumir um importante papel na promoção da qualidade do atendimento de bebês e crianças pequenas. A escala ITERS-R foi desenvolvida para ser utilizada nos Estados Unidos e, para a utilização da escala em outros países, é necessário todo um processo para sua validação.

Diante da possibilidade de contribuição da escala ITERS-R para avaliar a qualidade de creches inclusivas e mediante a necessidade de consolidação na prática do paradigma da inclusão, o objetivo deste trabalho foi verificar compreensão dos termos e a pertinência do conteúdo dos itens da Infant/Toddler Environment Rating Scale - Revised Edition que se referem às necessidades educacionais especiais, sob o referencial da psicometria.

\section{Método}

\section{Participantes:}

Participaram do estudo uma especialista em educação especial com domínio das línguas inglesa e portuguesa bem como conhecedora de ambas as culturas, que atuou no processo de tradução dos intens. Participaram sete alunos do curso de graduação em Psicologia da Universidade Federal de São Carlos no processo de análise semântica. Também participaram sete especialistas em educação especial e em educação infantil, todos vinculados ao Programa de Pós-Graduação em Educação Especial da Universidade Federal de São Carlos.

\section{Materiais, equipamentos e instrumentos:}

Foram utilizados materiais como papel, canetas, equipamentos como computador, impressora, gravador; foi utilizada uma cópia autorizada da escala ITERS-R (HARMS, CRYER; CLIFFORD, 2003), traduzida por Campos-de-Carvalho e colaboradores); protocolos para coleta de dados para os procedimentos de análise semântica e de análise de conteúdo. 


\section{Procedimento}

Foram selecionados os itens da escala ITERS-R (HARMS, CRYER; CLIFFORD, 2003) relacionados com a inclusão de crianças com necessidades educacionais especiais. O critério para seleção foi que o item deveria conter termos que remetessem ao atendimento às necessidades especiais, nas notas de esclarecimento ou nas questões. Foram selecionados 11 itens que tratavam explicitamente da inclusão de crianças com necessidades educacionais especiais, além de um item que se referia às necessidades educacionais especiais da equipe (Item 34). Assim, de um total de 39 itens, 12 itens da escala se referem à inclusão de pessoas com necessidades educacionais especiais. Foram eles: Espaço interno; Mobília para cuidados de rotina e brincadeira; Sono; Troca de fralda/toalete; Ajudando a criança a compreender linguage; Coordenação motora fina; Atividade física; Faz de conta; Promoção da aceitação da diversidade; Interação entre pares; Provisões para crianças com deficiências; Provisões para necessidades pessoais da equipe.

Estes itens foram traduzidos com a colaboração de uma especialista em Educação Especial, fluente em inglês e português, conhecedora das culturas americana e brasileira.

A partir da tradução dos itens selecionados para o estudo, foi realizada a verificação da compreensão dos termos da escala traduzida, baseado no procedimento de Análise Semântica. Esse procedimento tem como objetivo aferir a clareza/compreensão dos termos dos itens selecionados. A verificação da compreensão dos termos foi realizada por sete alunos do curso de Psicologia da Universidade Federal de São Carlos. Os alunos receberam instruções gerais sobre a escala e sobre o procedimento de análise semântica e efetuaram a análise tendo em mãos os itens traduzidos e os protocolos desenvolvidos para análise semântica, como os referido por Pasquali (2003). Todos os participantes assinaram o Termo de Consentimento Livre e Esclarecido.

Para verificação da pertinência do conteúdo, foi realizado procedimento baseado na Análise de Conteúdo (PASQUALI, 2003) utilizandose tabela de dupla entrada, desenvolvida para o estudo, para verificação da pertinência dos itens. Participaram do procedimento sete especialistas em Educação Especial e Educação Infantil, vinculadas ao Programa de PósGraduação em Educação Especial da UFSCar. Todas assinaram um Termo de Consentimento Livre e Esclarecido. A análise foi feita pelas especialistas tendo em mãos os itens reelaborados a partir da análise semântica e uma tabela de dupla entrada, como se refere Pasquali (2003). 


\section{Resultados}

Pode-se afirmar que nenhuma juíza mencionou a necessidade de exclusão de algum item, ou seja, todos foram pertinentes, ainda que os itens recebessem alguma observação e sugestões para que fosse mais acurado à cultura brasileira. Uma breve descrição do item e os principais comentários das especialistas e sugestões para melhoria são descritos a seguir.

O item Espaço Interno está relacionado com as condições do ambiente interno bem como o controle que o educador tem dele, como luminosidade e ventilação; são avaliados a amplitude do espaço, iluminação, temperatura, higiene do ambiente, conservação, acessibilidade. Uma especialista sugere que o espaço seja calculado de maneira mais precisa, em termos de metros quadrados por criança; outras, que a unidade de medida seja centímetro e não polegadas. Adicionalmente, indicaram a necessidade de verificar a presença de mofo para pontuar a qualidade do ambiente. Para pessoas com necessidades educacionais especiais, é um item adequado porque a acessibilidade já é requerida na pontuação "Mínimo" da escala, ou seja, para que a creche tenha um espaço interno minimamente adequado, há que ser adaptado para pessoas com deficiência, sejam elas crianças ou adultos frequentadores da creche. No entanto, a avaliação do espaço é considerada apenas no espaço da creche e a acessibilidade poderia ser avaliada antes mesmo da entrada da pessoa na instituição.

O item Mobília para Cuidados de Rotina e Brincadeira avalia a mobília para cuidados de rotina e as demais atividades a ser realizada com as crianças, relacionando o número de mobília, o estado de conservação, a adequação ao tamanho, tanto para crianças como para adultos, assim como acessibilidade. Avalia, também, o risco de ferimento em crianças devido a inadequações da mobília, a existência de lascas ou pregos expostos, por exemplo. Sugere-se acrescentar no item, para prevenir ferimento em crianças, que se evitasse a presença de quinas e cantos de móveis ou prever a devida proteção destas. Também seria importante descrever a inadequação de móveis sobrepostos ou perto da janela, nos quais a criança possa escalar porque há risco de queda; também poderia ser avaliada a roupa de cama ou berço das crianças para que se evitassem coberturas plásticas que, apesar de serem fáceis de limpar não permitem a absorção da transpiração da criança.

O item Disposição da Mobília avalia a relação dos móveis e dos espaços para outras atividades, acessibilidade dos espaços e acessibilidade dos materiais e possibilidade de fácil supervisão das crianças pelos educadores. Em relação às pessoas com necessidades educacionais especiais, a acessibilidade do espaço por meio do arranjo de mobília já está 
prevista na pontuação "Mínimo", ou seja, para o ambiente ser minimamente adequado, há que se dispor a mobília de maneira que permita o acesso de crianças e adultos com necessidades educacionais especiais.

O item Troca de fralda/toalete avalia, basicamente, a higiene e o faz em diferentes dimensões: a higiene do material, a higiene das crianças, a higiene dos educadores e a higiene do ambiente. Avalia também a qualidade das interações entre adultos e crianças nas situações de troca de fralda/ toalete e o ensino de habilidades de autocuidados. As observações das especialistas são em relação à imprecisão da expressão "Supervisão da criança de maneira inadequada ou não prazerosa" e a afirmação "Habilidades de autocuidados são ensinadas quando as crianças estão prontas" porque indicam que as crianças podem ser ensinadas, em diferentes níveis de exigência, mesmo quando são muito jovens. A indicação da escala para se cantar uma música enquanto se lava as maos é uma boa alternativa para tornar a atividade lúdica bem como para permanecer com o saponáceo tempo suficiente para que tenha ação de limpeza (durante 10 segundos). Sugere-se como alternativa à canção descrita na escala que está em inglês, a música "Lavar as mãos", de Arnaldo Antunes.

O item Ensinando a criança a compreender linguagem avalia a ajuda que as crianças recebem para compreender a linguagem. Enfoca a oportunidade e ocorrencia de interação verbal estimulantes entre educadoras e crianças, sinalizando a inadequação de a interação verbal ocorrer entre os adultos a despeito das crianças. As observações das especialistas foram em relação à dificuldade de avaliação, por exemplo, como avaliar a quantidade de conversa com bebês e crianças pequenas e a dificuldade de se aferir palavras como pouca, moderada, frequente é que fazem o observador a diferenciar a pontuação, por isso a necessidade de torná-las mais quantificáveis. Também questionaram o termo "Ajudando", apontando para a dificuldade de operacionalização do termo "quantidade de conversa moderada" bem como o quanto esta é significativa para a criança. Sugere-se que o item seja mais específico em relação à comunicação alternativa caso se verifique na creche crianças com deficiência auditiva, por exemplo.

O item Coordenação Motora Fina pretende avaliar as atividades de coordenação motora fina. O item avalia a adequação do material, o estado de conservação, a organização, acessibilidade assim com a rotatividade dos materiais e o nível de dificuldade que apresentam. Entretanto o item deixa de contemplar aspectos interacionais com os educadores e crianças, que sáo tao importantes para o desenvolvimento infantil. 
O item Brincadeira física ativa procura avaliar a oportunidade que o ambiente oferece para as crianças realizarem brincadeiras nas quais desenvolverão habilidades motoras, por isso os materiais e os espaços são avaliados, assim como a frequência de utilização dos espaços. Os exemplos de materiais contidos nas notas dos indicadores dão idéia do tipo de movimento que se espera que as crianças realizem para atender o item. Mas não são oferecidos exemplos de comportamentos que devem ser propiciados, como indicado pelas especialistas, como chutar, empurrar. As especialistas sugerem acrescentar exemplos para as diferentes faixas etárias das crianças. Além disso, mais uma vez o item deixa de considerar interação com a equipe. A intervenção do educador pode ser importante para desenvolver as habilidades motoras da criança por meio de brincadeiras físicas ativas. O educador pode oferecer os materiais, propor brincadeiras, apresentar atividades que desafiam as habilidades da criança e as estimulam. $O$ item também não prevê ou descreve brincadeiras físicas ativas que dispensam o uso de materiais, como brincadeiras tradicionais, que podem oferecer oportunidades de desenvolvimento motor em crianças pequenas - a história da creche no Brasil está relacionada com escassez de recursos financeiros e ausência de materiais. Assim, brincadeiras que estimulassem as habilidades motoras deveriam ser previstas neste item para que seja adequado à cultura brasileira; exemplos de brincadeiras em creches utilizando-se materiais de baixo custo ou sucata podem ser encontrados em Gil e Almeida (2001).

O item Faz-de-conta avalia os materiais oferecidos para as atividades, seja pela quantidade, acessibilidade e adequação ao tamanho: avalia a existência de materiais, a variedade e que estejam organizados de maneira que facilite o acesso da criança, mobílias do tamanho da criança. No entanto, as especialistas apontaram para a falta de ênfase do item na interação da equipe com as crianças, visto que só é abordada em um indicador no nível Excelente. Sugere-se que o item acrescente exemplos de material que represente tal condição, como óculos (sem lentes), óculos escuros, lupa, bengala. Adicionalmente, é importante mencionar que entre os exemplos de não constam sucata: blocos de madeira de diferentes tamanhos e espessuras, caixas de papelão, pedaços de tecido ou qualquer objeto que assuma a função representacional que a criança pretende em brincadeiras de faz-de-conta. Mais uma vez ressalta-se a importância da interação com a equipe, que é mencionada somente no último indicador da pontuação "Excelente". Sugere-se que o item contemple de maneira mais enfática a interação com a equipe a fim de estimular as crianças e propiciar a interação delas.

O item Promovendo aceitação da diversidade prevê que se os materiais apresentarem estereótipos negativos ou se a equipe mostrar preconceito em relação às pessoas com deficiência, o item deve ser pontuado 
como inadequado. A avaliação do preconceito é uma tarefa que exigiria do observador a habilidade de identificar a existência do preconceito na interação educador-criança, que muitas vezes ocorre de maneira tão sutil que se torna quase que imperceptível - mas não o é para aquele que está sendo discriminado. É necessário avaliar o preconceito na interação educadorcriança, uma vez que a maioria dos indicadores considera a diversidade em relação ao material mais do que em relação às interações. Para tratar de diversidade, o item faz referência à diversidade racial e cultural, de idade, de habilidade e de gênero assim como trata da diversidade apresentada sob o estereótipo negativo. Sugere-se tornar claro o que o item pretende ao considerar a diversidade para que este conceito seja único em todos os indicadores.

O item Interação entre pares avalia as condições oferecidas para que ocorra a interação entre pares assim como a intervenção das educadoras para propiciar interações adequadas em crianças. Conforme apontado pelas especialistas, o item poderia conter exemplos que tornassem objetivos termos como "interação negativa" e "interação positiva". Em relação a crianças com necessidades educacionais especiais, o item prevê a necessidade de se criar condições para a ocorrência de interações entre todas as crianças, inclusive crianças com deficiências, na pontuação 5, "Bom". Acrescentase que os colegas servem como modelo não só para a aprendizagem de utilização dos brinquedos, mas também para desempenho em atividades e habilidades. Esta consideração poderia contribuir para tornar o item ainda mais pertinente para avaliação da interação entre pares em relação a crianças com necessidades educacionais especiais. Sugere-se que a interação seja enfantizada e observada ao longo de toda a escala.

O item Provisão para crianças com deficiências avalia as necessidades da criança; o quanto a equipe busca atender as necessidades das crianças; as condições oferecidas para o envolvimento dos pais; a interação entre pares e atividades; as pequenas e grandes adaptações e modificações do ambiente; o envolvimento de profissionais especializados; a integração das crianças nas atividades e contribuição da equipe de trabalho para avaliações individuais e planos de intervenção. Exceto pelas diferenças em relação aos serviços de atendimento para crianças com deficiências que existem nos Estados Unidos e não existem no Brasil, as especialistas não fizeram nenhuma ressalva ou sugestão para o item. No entanto, o item só é aferido se existir uma criança com deficiência na sala e graus leves, médios de deficiência visual, auditiva ou mental, distúrbios de atenção ou problemas de aprendizagem nem sempre são facilmente identificáveis - menos ainda em crianças pequenas. $\mathrm{O}$ item ou a escala poderia deixar o observador sensível para estas situações, bem como considerar as crianças de risco. 
O item Provisões para necessidades pessoais da equipe enfoca a permanência de adultos com deficiência engajados na equipe de trabalho, o item considera a acessibilidade para estas pessoas na pontuação 3 "Mínimo", ou seja, para o ambiente ser avaliado como minimamente adequado, deve ser acessível para adultos com deficiência que trabalhem na creche e o ambiente deve ser pontuado como 5, "Bom", se houver acessibilidade existindo ou não pessoas com deficiências inseridos no programa. Assim, como atestaram as juízas, o item 34 mostra-se adequado para avaliar as provisões para membros da equipe com necessidades educacionais especiais.

\section{Discussão}

Por meio do estudo envolvendo os 12 itens da escala ITERS-R (HARMS, CRYER; CLIFFORD, 2003), infere-se que a escala é um mecanismo que contribui para se assegurar padrões mínimos de qualidade, necessidade apontada por documentos oficias (BRASIL, 1996; BRASIL, 1998). Assim é possível prevenir ou até mesmo impedir que a própria creche se torne um ambiente que implique em risco para problemas desenvolvimento e deficiência em crianças pequenas. A escala ITERS-R (HARMS, CRYER; CLIFFORD, 2003), porque pretende avaliar o atendimento a pessoas com deficiências, está de acordo com as políticas públicas brasileiras, estabelecidas por documentos oficiais (BRASIL, 1988; BRASIL, 1996; BRASIL, 1998; BRASIL, 2001), não porque discute políticas ou questões filosóficas a respeito da inclusão de pessoas com necessidades educacionais especiais, mas porque prevê a inclusão delas e o faz de maneira a deixar a avaliação objetiva podendo captar diferentes estágios que a creche pode estar em relação ao processo de inclusão, assim como indica, nas pontuações "Bom" e "Excelente" o que se espera do atendimento de pessoas com necessidades educacionais especiais. Pode-se verificar também que a escala está de acordo com Marques (2000) porque prevê o atendimento de crianças pequenas com necessidades educacionais especiais orientado para educação e o cuidado. Verifica-se nos itens da escala ITERS-R (HARMS, CRYER; CLIFFORD, 2003) a importância do caráter educativo do ambiente considerado adequado para as crianças, por meio da estimulação da linguagem, autocuidados, desenvolvimento de coordenação motora e enriquecimento das experiências da criança.

\section{Conclusão}

A pertinência dos itens, indicada pelas juízas especialistas em educação especial, sugere que os itens podem ser úteis para se avaliar creches que atendem crianças com deficiência, assim como para explicitar o que se espera do atendimento em creche para esta população. Indica-se a necessidade de novos estudos para estudar a validade da escala para 
o contexto brasileiro, porque esta poderia servir como instrumento para responder às necessidades de promoção da qualidade do atendimento de crianças com necessidades especiais em creches.

Referências

ANTUNES, A. Lavar as mãos. Castelo Rá-tim-Bum, faixa 13. Velas, 1995, CD.

ABRAMOWICZ, A, Rocha, M. J. S, Cunha, I. A. M., O desenvolvimento das crianças de três a seis anos. In: PALHARES, M.S, MARINS, S. Escola Inclusiva. São Carlos, Edufscar, 2002.

BRASIL. Constituição. Constituição [da] Republica Federativa do Brasil. Brasília: Senado Federal, 1988.

Estatuto da criança e do adolescente. São Paulo: Cortez, 1990.

Lei de Diretrizes e Bases da Educação Nacional n. 9.394 de 20 de dezembro de 1996. Diário oficial da União, Brasília, 21 de dezembro de 1996.

. Ministério da Educação e Cultura. Referencial Curricular Nacional para a Educação Infantil. Brasília, DF, 1998.

Ministério da Educação e Cultura. Diretrizes Nacionais para a Educação Especial na Educação Básica, DF, 2001.

CAMPOS, M. M.; ROSEMBERG, F. Critérios para um atendimento em creches que respeite os direitos fundamentais das crianças. Brasília: MEC, SEB, 2009, 44 p.

DAHLBERG, G. MOSS, P. \& PENCE, A. Qualidade na educação da Primeira Infância: perspectivas pós-modernas. Porto Alegre, ArtMed, 2003.

FERREIRA, J. R., MENDES, E. G., NUNES, L. R. D. P., GLAT, R. Integração/ Inclusão. Relatório enviado à FAPESP, não publicado, 2001.

GIL, M.S.C.A.; ALMEIDA, N.V.F. Brincando na creche. 1. ed. São Carlos: EdUFSCar, 2001.

HARMS, T.; CLIFFORD, R. M \& CRYER, D. R. Early Childhood Environment Rating Scale- Revised Edition. New York: Teachers College Press, 2003. 
MARQUES, L. P. Educação infantil inclusiva: um desafio possível. Temas sobre Desenvolvimento, v. 8, n. 48, p. 30-7, 2000.

MENDES, E. G. Perspectivas para a Construção da Escola Inclusiva no Brasil. In: Palhares, M. S. e Marins, S. Escola Inclusiva, São Carlos, Edufscar, 2002.

MENDES, E. G.; FERREIRA, J. R.; NUNES, L. R. D. P.; GLAT, R. PRODISC IV- Análise crítica das teses e dissertações sobre Educação Especial nas áreas de Educação e Psicologia. Relatório I, Processo FAPESP n. 2000/07485-0, 2001.

MINTO, C. A. Educação Especial: Da LDB aos Planos Nacionais de Educação - Mec e Proposta da Sociedade Brasileira. In: Palhares, M. S. e Marins, S. Escola Inclusiva, Edufscar, São Carlos, 2002.

PASQUALI, L. Psicometria - Teoria dos testes na Psicologia e na Educação. Vozes: Petrópolis, 2003.

PIOTTO, D.C., CHAGURI, A.C, MELLO, A.M., SILVA, A.P.S. ELTNK, C., YAZLE, C.H., CARNIEL, I.C., SORDI, G., BALDIN, L.S.A., FREDERICK, M.I.B., MORAIS, R. BESANI, V. \& ROSSETI-FERREIRA, M.C. Promoção de qualidade e avaliação na educação infantil: uma experiência. Cadernos de pesquisa, n. 105, p. 53-76, 1998.

ROSA, L. C. S. Formação continuada de atendentes para inclusão de crianças com necessidades educacionais especiais em creches. Dissertação de mestrado. São Carlos: PPGEES/UFSCar, 2003.

ROSSetTI-FERREIRA M. C., RAMON, F. \& SILVA, A. P. S. Políticas de atendimento à criança pequena nos países desenvolvidos. Cadernos de pesquisa, n. 115, São Paulo, março de 2002.

\section{Correspondência}

Lisandrea Rodrigues Menegasso Gennaro - Rodovia Washington Luís, km 235, 310 - CEP 13565-905 - São Carlos, São Paulo, Brasil.

E-mail: lisandrea@gmail.com - mscagil@ufscar.com.br 